\title{
Heme oxygenase-1 attenuates inflammation and oxidative damage in a rat model of smoke-induced emphysema
}

\author{
JINGJING WEI ${ }^{1,3^{*}}$, GUOQUAN FAN ${ }^{2 *}$, HUI ZHAO ${ }^{3}$ and JIANQIANG $\mathrm{LI}^{3}$ \\ Departments of ${ }^{1}$ Pediatrics and ${ }^{2}$ Microbiology and Immunology, Shanxi Medical University; ${ }^{3}$ Department of Critical Care \\ and Pulmonary Medicine, Shanxi Medical University Second Hospital, Taiyuan, Shanxi 030001, P.R. China
}

Received April 3, 2015; Accepted September 7, 2015

DOI: $10.3892 /$ ijmm.2015.2353

\begin{abstract}
Emphysema is a serious disease of the respiratory system and is associated with inflammation and oxidative stress. Heme oxygenase-1 (HO-1), a rate-limiting enzyme involved in heme biosynthesis, exerts potent anti-inflammatory, antioxidant, anti-apoptotic and anti-proliferative effects in various diseases. In the present study, we examined the effects of HO-1 on smoke-induced emphysema, as well as the underlying mechanisms in a rat model of smoke-induced emphysema. Rats were either exposed to cigarette smoke or sham-exposed for 20 weeks to establish the model of smoke-induced emphysema. The rats were subcutaneously injected with protoporphyrin IX [tin-protoporphyrin IX (SnPP) or ferriprotoporphyrin IX chloride (hemin)] during this period to examine the protective effects of HO-1. Subsequently, the development of emphysema, inflammatory cells, the levels of inflammatory mediators, particularly interleukin (IL)-17, tumor necrosis factor (TNF)- $\alpha$, monocyte chemotactic protein-1 [MCP-1, also known as chemokine (C-C motif) ligand 2 (CCL2)], IL-8 [also known as chemokine (C-X-C motif) ligand 8 (CXCL8)], macrophage inflammatory protein- $2 \alpha$ [MIP- $2 \alpha$, also known as chemokine (C-X-C motif) ligand 2 (CXCL2)] and IL-10, as well as the malondialdehyde (MDA), superoxide dismutase (SOD) and glutathione (GSH) content were determined. Exposure to smoke increased the total cell, neutrophil and macrophage counts in the bronchoalveolar lavage fluid (BALF). It also
\end{abstract}

Correspondence to: Dr Jianqiang Li, Department of Critical Care and Pulmonary Medicine, Shanxi Medical University Second Hospital, 382 Wuyi Road, Taiyuan, Shanxi 030001, P.R. China E-mail: ljqhx@sina.com

Dr Jingjing Wei, Department of Pediatrics, Shanxi Medical University, 56 Xinjian Road, Taiyuan, Shanxi 030001, P.R. China

E-mail: weijj82@126.com

${ }^{*}$ Contributed equally

Key words: chronic obstructive pulmonary disease, bilirubin, emphysema, inflammation, oxidative stress, heme oxygenase-1, interleukin-17 increased the levels of the inflammatory mediators, IL-17, TNF- $\alpha$, MCP-1, IL- 8 and MIP- $2 \alpha$, as well as the MDA content and induced emphysema. Treatment with hemin upregulated HO-1 expression and attenuated the development of smokeinduced emphysema by reducing inflammatory cell infiltration, decreasing the levels of inflammatory mediators and attenuating oxidative damage, to a certain extent. In conclusion, our findings demonstrate that $\mathrm{HO}-1$ exerts anti-inflammatory and antioxidant effects, thus attenuating the development of smokeinduced emphysema.

\section{Introduction}

Chronic obstructive pulmonary disease (COPD) is characterized by chronic inflammation and oxidative damage that results in airflow limitation; these changes are not fully reversible and the disease is associated with progressive development. Emphysema is the main pathological change and the major factor responsible for the decline in lung function in COPD. In recent years, COPD has become a major global public health concern and is associated with high morbidity and mortality (1). Currently, smoking is widely recognized as a major risk factor for COPD. Cigarette smoke contains several harmful chemicals that may lead to inflammatory responses and oxidative stress in airway epithelial cells, fibroblasts and alveolar epithelial cells. Inflammatory and alveolar epithelial cells release reactive oxygen species (ROS), which stimulate inflammatory cells to release inflammatory mediators. The interactions between these mediators promote the development of COPD (1-4).

Inflammatory cells, such as neutrophils, alveolar macrophages and $\mathrm{T}$ lymphocytes are activated by stimuli in vivo and release a variety of inflammatory mediators, such as leukotriene B4 (LTB4), tumor necrosis factor (TNF)- $\alpha$, interleukin (IL)-17 and chemokines, such as IL-8 [also known as chemokine (C-X-C motif) ligand 8 (CXCL8)] (1-5). These mediators can damage the lung structure, promote neutrophil inflammation and play an important role in the inflammation and pathogenesis of COPD. IL-8, macrophage inflammatory protein-2 $\alpha$ [MIP-2 $\alpha$, also known as chemokine (C-X-C motif) ligand 2(CXCL2)] and monocyte chemotactic protein-1 [MCP-1, also known as chemokine (C-C motif) ligand 2 (CCL2)] are chemokines, that recruit neutrophils and monocytes to sites of inflammation (6-10). Thus, these inflammatory mediators play an important role in the development of emphysema. 
Heme oxygenase-1 (HO-1) is a rate-limiting enzyme involved in mammalian heme biosynthesis. HO-1 breaks down heme to form equimolar amounts of biliverdin, free iron and carbon monoxide $(\mathrm{CO})$ and it is rapidly activated by its physiological substrate, heme, as well as inflammation and oxidative stress-related stimuli, nitric oxide (NO), heat shock and other factors. HO-1 and its products exert potent anti-inflammatory, antioxidant, anti-apoptotic and anti-proliferative effects under several and pathologies, such as asthma, organ transplantation, acute lung injury (11-17).

Studies have found that HO-1 plays a role in the development of COPD characterized by chronic inflammation and oxidative damage and the overexpression of HO-1 mediated by adenovirus in the lungs attenuates the development of elastase-induced pulmonary emphysema in mice (18-27). We thus hypothesized that the upregulation of HO-1 expression induced by hemin may prevent the development of emphysema by inhibiting inflammation and the associated damage induced by oxidative stress.

\section{Materials and methods}

Animal model. Healthy male Wistar rats (4 weeks of age, weighing 100-120 g) were obtained from the Experimental Animal Center, Chinese Academy of Military Science. The present study was approved by the Institutional Review Board for Biomedical Research of Shanxi Medical University, Taiyuan, China. The rats were allowed to acclimatize for 2 weeks and had free access to food and water. The rats were then randomly divided into 5 groups ( $\mathrm{n}=10 /$ group) as follows: i) the control group (C): normal saline (NS) + sham smoke; ii) the smokeexposed group (SM): NS + smoke; iii) the hemin group $(\mathrm{H})$ : hemin + smoke; iv) the SnPP group (S): SnPP + smoke; and v) the $\mathrm{H}+\mathrm{S}$ group (HS): hemin $+\mathrm{SnPP}+$ smoke. For 20 weeks, the rats were treated with $0.3 \mathrm{ml} \mathrm{NS}$ or $0.3 \mathrm{ml}$ protoporphyrin IX [tin-protoporphyrin IX (SnPP), an inhibitor of HO-1 activity or ferriprotoporphyrin IX chloride (hemin), an inducer of HO-1] once daily for the first 3 days of each week and exposed to smoke (or sham smoke) once daily from the 2nd to the 6th day of each week. After 20 weeks, the animals were sacrificed, as previously described (28).

The smoking regimen was 16 cigarettes each day, each for $10 \mathrm{~min}$, with an interval of $5 \mathrm{~min}$ between cigarettes. The rats were exposed to 24 puffs of cigarette smoke from 2 cigarettes in a smoke exposure device (PAB-S200; Beijing Biolaunching Technologies Co., Ltd., Beijing, China). The Monkey King brand of cigarettes were commercial filter cigarettes produced by the Baoji Cigarette Factory, Baoji, China and had a cigarette standard tar volume of $11 \mathrm{mg}$.

Hemin or SnPP (Frontier Scientific, Inc., Logan, UT, USA) were dissolved in $1 \mathrm{M} \mathrm{NaOH}$ and adjusted to $\mathrm{pH}$ 7.3-7.5 with $\mathrm{HCl}$ and then diluted to the final concentration with NS. The rats were subcutaneously injected with hemin $(20 \mu \mathrm{mol} / \mathrm{kg})$ or $\mathrm{SnPP}(20 \mu \mathrm{mol} / \mathrm{kg})$, or hemin $(20 \mu \mathrm{mol} / \mathrm{kg})+\operatorname{SnPP}(20 \mu \mathrm{mol} / \mathrm{kg})$ or NS. The dose of hemin used in the present study was decided upon following testing in preliminary experiments (data not shown) and examing doses used in a previous study (41). In a previous study (41) the dose of hemin used was $75 \mu \mathrm{mol} / \mathrm{kg}$. However, we found that this dose was too high for long-term use, and from our preliminary experiments, we found that the dose of $20 \mu \mathrm{mol} / \mathrm{kg}$ hemin was the opitam one for long-term use.

Collection of bronchoalveolar lavage and other specimens. The animals were anesthetized and 8-10 ml blood specimens were drawn from the abdominal aorta. The trachea was cannulated, the right lung was ligated and the left lung was intubated and injected thrice with $2 \mathrm{ml}$ pre-cooled saline $\left(4^{\circ} \mathrm{C}\right)$. Bronchoalveolar lavage fluid (BALF) was recovered and centrifuged. The supernatant was stored at $-80^{\circ} \mathrm{C}$ and the cells were allowed to settle, stained with Wright's dye (dye includes methylene blue and eosin Y dissolved in methyl alcohol; all obtained from Beyotime Institute of Biotechnology, Shanghai, China) and counted using a standard technique. The right upper lung tissue was removed, fixed with formalin, embedded in paraffin, sectioned and stained with hematoxylin and eosin [(H\&E), H\&E staining kit (C0105; Beyotime Institute of Biotechnology, Shanghai, China)]. The pathological changes of the lung tissues were then observed at x10 and x40 magnification under a light microscope. The lung, BALF supernatant and serum were stored at $-80^{\circ} \mathrm{C}$ for other measurements.

Cell count in BALF. Following the recovery of BALF and placed in a hemocytometer. The number of cells counted was the sum of all cells across 4 squares in one chamber. The number of cells was calculated using the following formula: total number of cells $/ \mathrm{ml}=$ cells in 4 squares $/ 4 \times 10^{4}$ ). The remaining BALF was centrifuged at $400 \mathrm{x} \mathrm{g}$ at $4^{\circ} \mathrm{C}$ for $5 \mathrm{~min}$. The supernatant was saved and the precipitated cells were smeared and stained with Wright's staining solution. The total number of cells, macrophages and neutrophils was then counted in 5 high-power (x40 magnification) microscopic fields, and we then calculated the mean percentage of macrophages (or neutrophils) in the total cells and the numbers $/ \mathrm{ml}=$ the mean percentage $\mathrm{x}$ the total number of cells $/ \mathrm{ml}$.

Morphometric evaluation of emphysema. Alveolar airspace enlargement was assessed according to the mean linear intercept (MLI), mean alveolar number (MAN) and mean alveolar area (MAA) by 2 independent individuals in a blinded manner, as previously described (29). MLI involved centering a 'cross' on the middle of each field, counting the number of alveolar septa (Ns) through the crosshairs and measuring the total length of the crosshairs (L), MLI = L/Ns. MAN was calculated by counting the number of alveoli $(\mathrm{Na})$ within each field divided by the area of the field (TA), MAN $=\mathrm{Na}$ /TA. MAA involved counting the number of alveoli $(\mathrm{Na})$ within each field, measuring the area of each field(TA) and H\&E staining (lung parenchyma) of the area $(\mathrm{PA}), \mathrm{MAA}=(\mathrm{TA}-\mathrm{PA}) / \mathrm{Na}$.

Preparation of $10 \%$ lung tissue homogenates. The rat lung tissue $(100 \mathrm{mg}$ ) was accurately weighed, cut into small pieces and placed into 9 volumes of normal saline by weight $(\mathrm{g}) /$ volume $(\mathrm{ml})=1: 9$, and homogenized using an ultrasonic processor (CP-750, Cole-Parmer North America, Vernon Hills, IL, USA). The supernatant was isolated by centrifugation at 3,000 rpm for $10 \mathrm{~min}$ and stored at $-80^{\circ} \mathrm{C}$ for later use in other analyses.

Measurement of cytokine levels. In accordance with the instructions provided with the ELISA kit (Ebioscience, San Diego, 
CA, USA), the levels of cytokines (TNF- $\alpha$ and IL-8 in serum and BALF, IL-17, IL-10, MIP-2 $\alpha$ and MCP-1 in serum, BALF and lung tissue supernatant) were measured. The OD values were measured using a microplate reader (Gemini EM, Molecular Devices, Sunnyvale, CA, USA) at $450 \mathrm{~nm}$. Standard curves were created and the concentrations were calculated according to the relevant formulas.

Immunohistochemistry (IHC). Tissue blocks were formalin-fixed, paraffin-embedded, sectioned, de-paraffinized in 3 changes of xylene ( 5 min each), hydrated in 2 changes of $100 \%$ ethanol (10 min each) and subsequently hydrated in 2 changes of $95 \%$ ethanol (10 min each). Antigen unmasking was performed in a pressure cooker with $10 \mathrm{mM}$ sodium citrate buffer, $\mathrm{pH}$ 6.0. Endogenous peroxidase activity was quenched by placing the sections in a $1 \%$ hydrogen peroxide solution in methanol for $30 \mathrm{~min}$. The tissue sections were then washed and immunostaining was performed using a commercially available SABC (streptavidin biotinylated complex) kit (SA1025, Boster, Wuhan, China) according to the manufacturer's instructions. The tissue sections were stained with HO-1 antibody (ab13248; Abcam, Cambridge, MA, USA). Images of the stained sections were digitally captured at $\mathrm{x} 40$ magnification using a Nanozoomer system (Olympus American Inc., Center Valley, PA, USA) and the average gray value of staining was measured on each image by one of the investigators in a blinded manner. The average gray value was negatively-related to the depth of protein staining.

Western blot analysis. Whole lung tissue $(50 \mathrm{mg})$ was accurately weighed, cut into small pieces and placed into $500 \mu 1$ RIPA lysis buffer (Beyotime Institute of Biotechnology, Haimen, China) and homogenized using an ultrasonic homogenizer on ice. The supernatant was isolated by centrifugation at $12,000 \mathrm{rpm}$ for $10 \mathrm{~min}$ and stored at $-80^{\circ} \mathrm{C}$ for use in western blot analysis. $\beta$-actin and HO-1 protein expression was then measured by western blot analysis. Proteins were separated according to their molecular weight and blotted onto PVDF membranes. The membranes were blocked for $2 \mathrm{~h}$ in 5\% BSA and incubated with rabbit-anti-rat HO-1 antibody (1/1,000, H-105; Santa Cruz Biotechnology, Inc., Santa Cruz, CA, USA) followed by a peroxidase labeled goat anti-rabbit antibody $(1 / 2,000$, BA1055; Boster). For the protein loading control, the membrane was stripped using a $25 \mathrm{mM}$ glycine- $\mathrm{HCl}$ buffer containing $1 \%$ SDS and incubated with mouse anti-rat $\beta$-actin antibody $(1 / 1,000$, BM0626; Boster) followed by a peroxidase labeled goatanti-mouse antibody (1/2,000, BA1050; Boster). The bands of interest were visualized by enhanced chemiluminescence kit (AR1111, Boster) according to the protocol in provided with the FluorChem HD2 System (Protein Simple, San Jose, CA, USA). The relative protein expression levels of HO- $1 / \beta$-actin were then obtained by analyzing the OD value ratios of the bands with Image-Pro Plus 6.0 software (Media Cybernetics, Inc., Rockville, MD, USA).

Malondialdehyde (MDA), superoxide dismutase (SOD) and glutathione (GSH) assays. The tissue specimens were allowed to naturally thaw to room temperature. The OD values representing the MDA levels in lung homogenates and serum were measured at $532 \mathrm{~nm}$ according to the manufacturer's instructions provided with the TBA kit (Jiancheng Biotech Co., Ltd., Nanjing, China). The OD values representing the SOD levels in the lung tissue supernatant and serum were measured at $550 \mathrm{~nm}$ according to the manufacturer's instructions provided with the hydroxylamine kit (Jiancheng Biotech Co., Ltd). The OD values representing the GSH levels in the lung tissue supernatant and serum were measured at $420 \mathrm{~nm}$ according to the manufacturer's instructions provided with the reduced glutathione assay kit (Jiancheng Biotech Co., Ltd). The concentrations of MDA, SOD and GSH were then calculated according to the instructions provided with the respective kits.

Statistical analysis. The results are expressed as the means \pm SD. Statistical analysis was performed using SPSS 17.0 software and two-way analysis of variance (ANOVA) was used to assess the differences between groups. A P-value $<0.05$ was considered to indicate a statistically significant difference.

\section{Results}

Effects of treatment with hemin and/or SnPP and exposure to cigarette smoke on the expression levels of $\mathrm{HO}-1$. The results of IHC and western blot analysis revealed that compared with the control (C) and smoke-exposed (SM) groups, treatment with hemin (an inducer of HO-1), SnPP (an inhibtor of HO-1 activity) or SnPP + hemin (H, S and HS groups) resulted in a significant increase in HO-1 protein expression in the lungs, particularly in alveolar macrophages. In IHC, this increase in HO-1 expression was evidenced by an increase in the amount of dark brown staining. Exposure to cigarette smoke (SM group) also resulted in a small increase in HO-1 expression (Fig. 1).

Exposure to cigarette smoke induces pathological changes associated with emphysema and these changes are attenuated by treatment with hemin. Cigarette smoke-induced emphysema was observed after 20 weeks of exposure to cigarette smoke, evidenced by pathological changes associated with emphysema (alveolar airspaces expanded universally and integrated into a larger cavity, alveolar pores expanded and many alveolar septa became narrow and broken). Treatment with hemin (inducer of HO-1; $\mathrm{H}$ group) attenuated these pathological changes; however, treatment with SnPP (an inhibitor of HO-1 activity; $\mathrm{S}$ group) did not reverse the adverse effects of smoke (Fig. 2). The development of emphysema was also evidenced by a significant increase in the MLI and MAA values and a significant decrease in the MAN values in the rats exposed to cigarette smoke (SM group) compared to the control rats (C), indicating alveolar airspace enlargement. Treatment with hemin partly reversed these effects, decreasing the MLI and MAA values and increasing the MAN values (the values did not completely return to those of the controls though). However, treatment with SnPP increased the MLI and MAA values and decreased the MAN values (Fig. 3). These results demonstrate that the activation of HO-1 attenuates the adverse effects of cigarette smoke, preventing alveolar airspace enlargement.

Exposure to cigarette smoke results in an increase in inflammatory cell infiltration and elevated levels of inflammatory mediators and these effects are partly reversed by treatment with hemin. Exposure to cigarette 

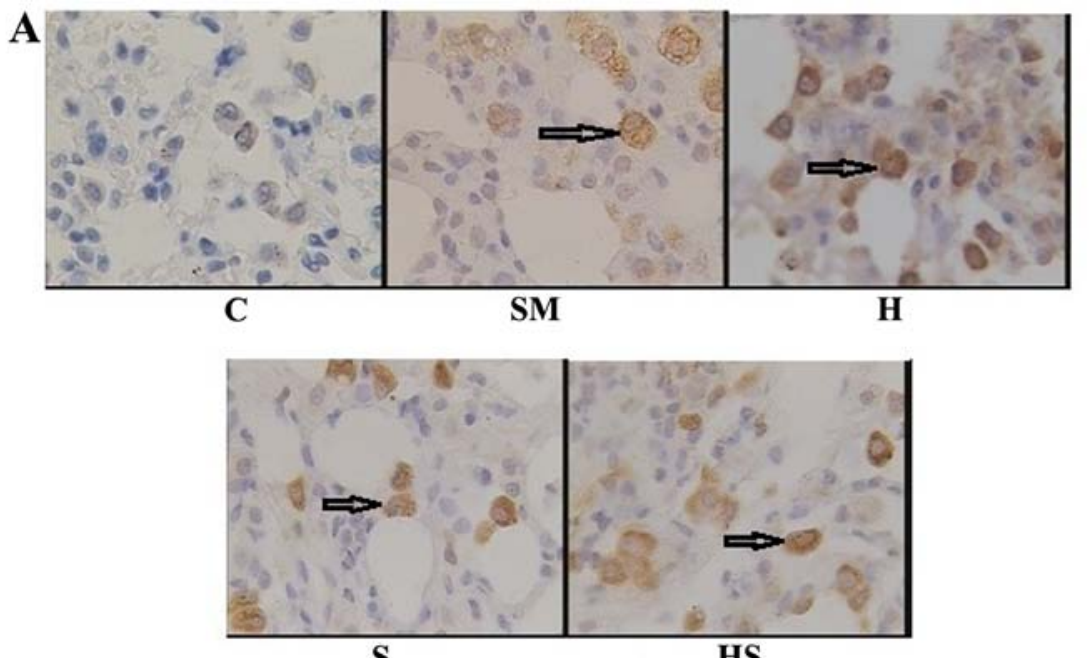

S

HS

B

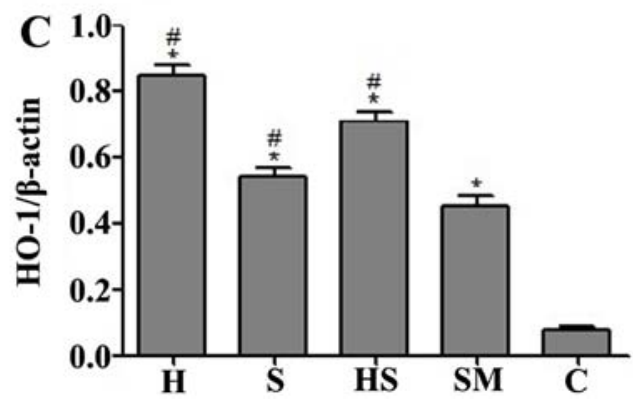

Figure 1. Heme oxygenase-1 (HO-1) protein expression. Hemin or hemin + SnPP treatment and cigarette smoke exposure upregulated the expression of HO-1. (A) Representative IHC images of HO-1 expression (dark brown) in lung tissues shown for each group (x40 magnification). Arrows indicate macrophages. (B) Protein bands for $\beta$-actin (loading control) and HO-1 detected by western blot analysis following long-term smoke exposure and protoporphyrin treatment. (C) Quantitative analysis of the relative protein expression levels of HO- $1 / \beta$-actin. " $\mathrm{P}<0.05$, compared with the control (C) group; " $\mathrm{P}<0.05$, compared with the smoke-exposed (SM) group. C, sham smoke; SM, cigarette smoke; H, hemin + smoke; S, SnPP + smoke; HS, hemin + SnPP + smoke.

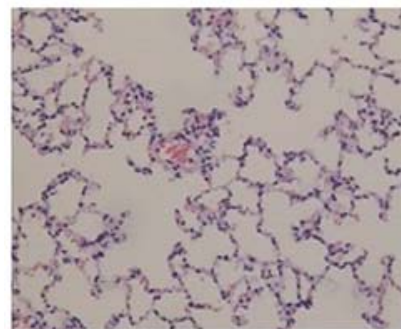

C

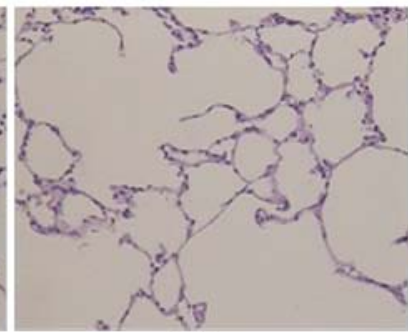

SM

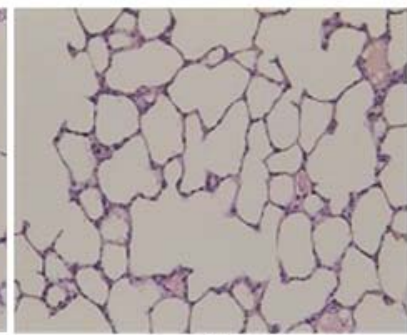

H

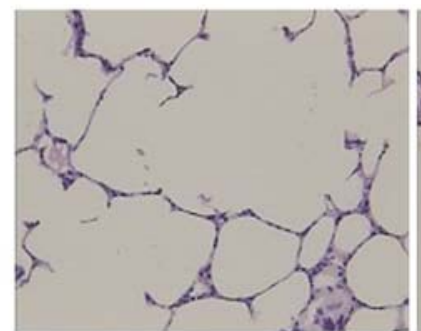

S

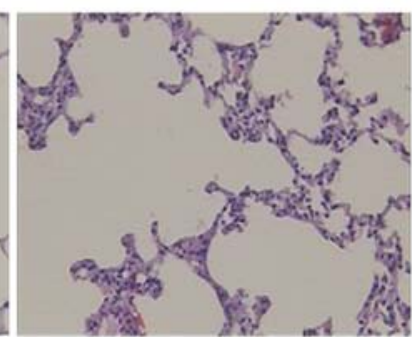

HS

Figure 2. Pathological changes in lung tissue. A representative pathological image is shown for each group (H\&E, x10 magnification). Cigarette smoke exposure induced pathological changes associated with emphysema (SM group) and hemin treatment reduced pulmonary injury (H and HS groups). In the control (C) group, alveolar distribution was uniform and alveolar septa were complete. Alveolar airspaces expanded universally and integrated into a larger cavity, alveolar pores expanded, and many alveolar septa became narrowed and broken in the SM, S and HS groups, whereas these changes were alleviated in the H group. C, sham smoke; SM, cigarette smoke; H, hemin + smoke; S, SnPP + smoke; HS, hemin + SnPP + smoke.

smoke (SM group) induced an increase in inflammatory cell infiltration; the total cell, neutrophil and macrophage counts in BALF were significantly higher in the SM group than those in the control group (C) (Fig. 4). Treatment with 

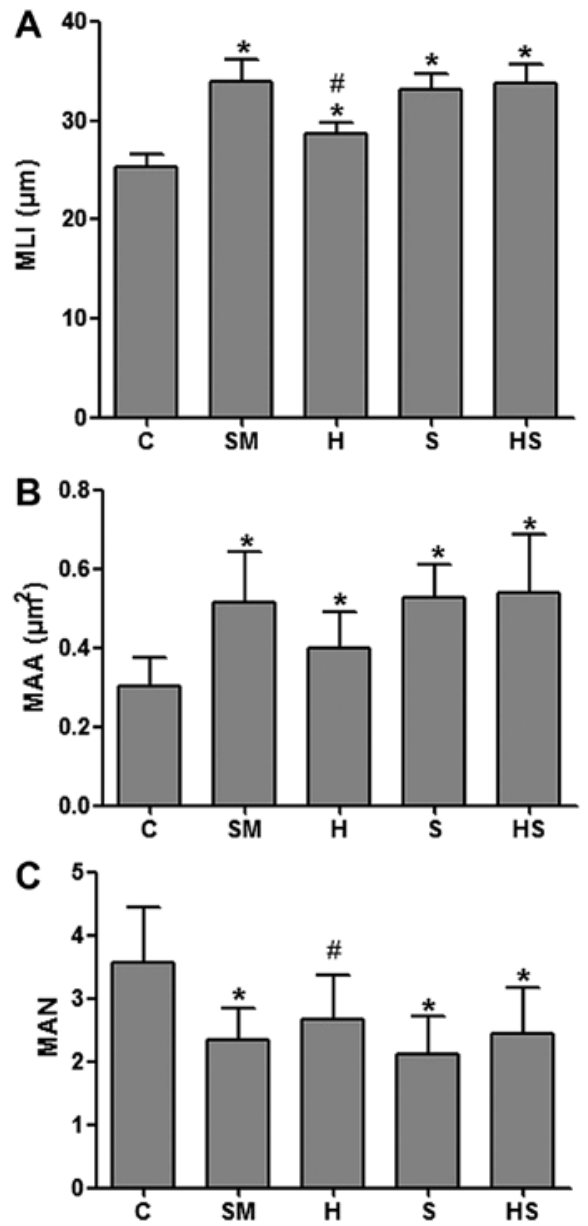

Figure 3. Morphometric evaluation of emphysema following long-term cigarette smoke exposure and protoporphyrin treatment. Long-term cigarette smoke exposure induced significant changes in (A) MLI, (B) MAA and (C) MAN values and hemin treatment attenuated these pulmonary morphometric changes. ${ }^{*} \mathrm{P}<0.05$ compared with the control $(\mathrm{C})$ group; ${ }^{\text {}} \mathrm{P}<0.05$ compared with the smoke-exposed (SM) group. C, sham smoke; SM, cigarette smoke; H, hemin + smoke; S, SnPP + smoke; HS, hemin + SnPP + smoke.

hemin (inducer of HO-1; $\mathrm{H}$ group) reduced inflammatory cell infiltration to a certain extent (shown by the decrease in the total cell, neutrophil and macrophage count in BALF). However, treatment with SnPP (an inhibitor of HO-1; S group) did not reverse the adverse effects of smoke; the total cell and neutrophil counts significantly increased compared to the control (C group) (Fig. 4). Exposure to cigarette smoke also significantly increased the levels of the inflammatory mediators, including IL-17, TNF- $\alpha$, IL-8, MCP-1 and MIP- $2 \alpha$. The TNF- $\alpha$ and IL- 8 levels significantly increased in the serum and BALF in the SM group compared to the control group. Treatment with hemin significantly reduced these effects. The IL-17 levels significantly increased in the BALF and lung tissue following exposure to cigarette smoke. Treatment with hemin decreased these levels in the BALF and lung tissue. SnPP increased the IL-17 levels in the BALF and lung tissue. The MIP- $2 \alpha$ levels increased in the lung tissue, serum and BALF following exposure to cigarette smoke, and treatment with hemin decreased these levels. However, in the SnPP-treated group, the MIP- $2 \alpha$ levels increased. The IL-10 levels significantly decreased in the serum of the smokeexposed rats. Treatment with hemin significantly increased the

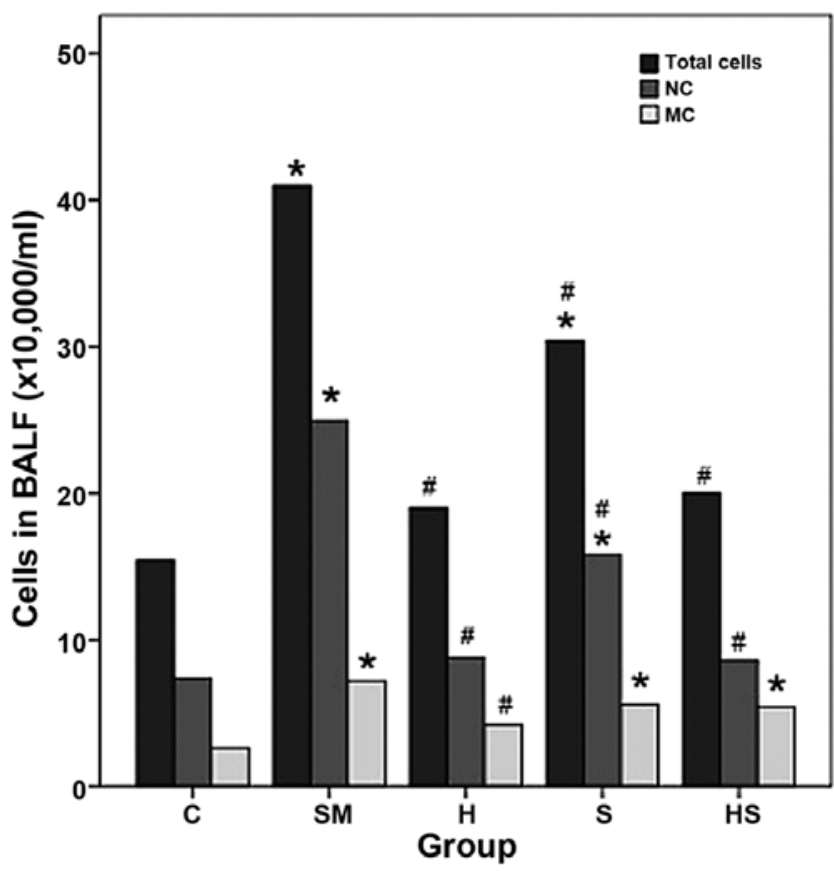

Figure 4. Total cell, neutrophil (NC) and macrophage counts (MC) in BALF. Cigarette smoke exposure induced increases in the infiltration of these inflammatory cells and hemin treatment suppressesed this infiltration. ${ }^{*} \mathrm{P}<0.05$ compared with the control $(\mathrm{C})$ group; ${ }^{\text {"}} \mathrm{P}<0.05$ compared with the smoke-exposed (SM) group. BALF, bronchoalveolar lavage fluid; C, sham smoke; SM, cigarette smoke; H, hemin + smoke; S, SnPP + smoke; HS hemin + SnPP + smoke.

IL-10 levels in the serum, BALF and lung tissue compared to the smoke-exposed rats. SnPP had no significant effect on the IL-10 levels. The levels of MCP-1 significantly increased in the serum, BALF and lung tissue in the SM group compared to the control group. Hemin significantly decreased these levels compared to the SM group. Treatment with SnPP also increased the MCP-1 levels compared to the controls. The levels of the inflammatory mediators did not completely return to those of the controls, indicating that hemin partly suppressed inflammation induced by cigarette smoke (Fig. 5).

Treatment with hemin decreases the MDA, and increases the SOD and GSH contents. Following exposure to cigarette smoke (SM group), the MDA levels in the serum and lung tissue homogenate were higher than those in the sham smoke (control, C) group $(\mathrm{P}<0.05)$, and the SOD levels in the lung tissue in the SM group were lower than those in the control group $(\mathrm{P}<0.05)$. Treatment with hemin decreased the MDA, and increased the SOD and GSH contents. These results indicate that HO-1 exerts antioxidant effects in rats exposed to cigarette smoke (Fig. 6).

\section{Discussion}

In the present study, we found that long-term treatment with hemin significantly upregulated $\mathrm{HO}-1$ protein expression, reduced the levels of inflammatory cytokines and chemokines and the infiltration of inflammatory cells, inhibited oxidative stress-induced damage, and protected the lungs from the development of pathological changes associated with emphysema. 

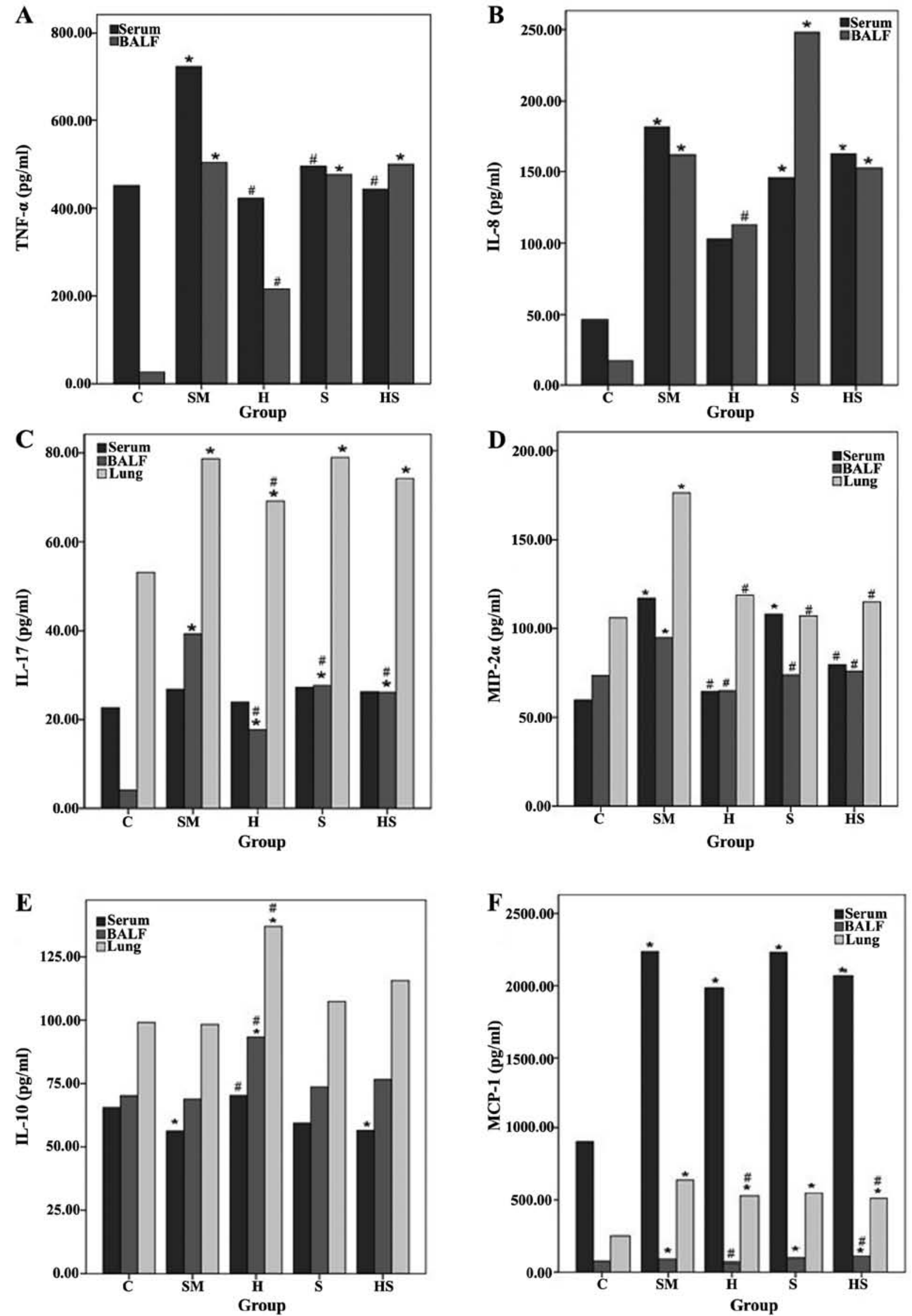

Figure 5. (A-F) Levels of inflammatory cytokines and chemokines in serum, BALF and lung tissue. Cigarette smoke exposure significantly increased the levels of the inflammatory mediators, such as IL-17, TNF- $\alpha$, IL-8, MCP-1 and MIP-2 $\alpha$, and hemin treatment reduced the levels of these mediators, except for IL-10. ${ }^{*} \mathrm{P}<0.05$ compared with the control (C) group; ${ }^{*} \mathrm{P}<0.05$ compared with the smoke-exposed (SM) group. BALF, bronchoalveolar lavage fluid; C, sham smoke; SM, cigarette smoke; H, hemin + smoke; S, SnPP + smoke; HS, hemin + SnPP + smoke. 

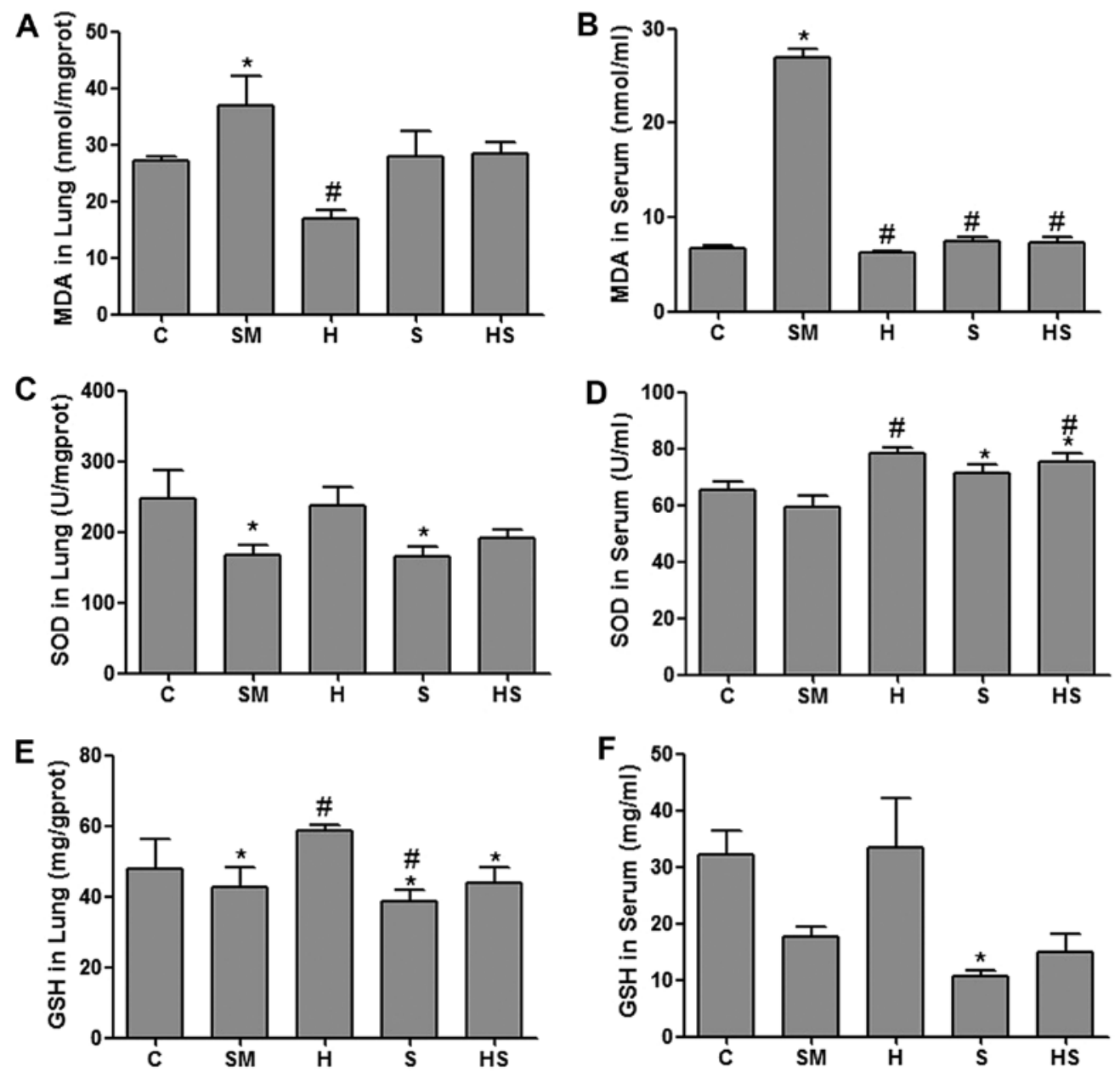

Figure 6. Oxidative damage and antioxidant levels in lung tissues and serum. Hemin treatment reduced the MDA and increased the SOD and GSH contents following exposure to cigarette smoke. (A) MDA levels in lung tissue. (B) MDA levels in serum. (C) SOD levels in lung tissue. (D) SOD levels in serum. (E) GSH levels in lung tissue. (F) GSH levels in serum. ${ }^{*} \mathrm{P}<0.05$ compared with the control (C) group; ${ }^{*} \mathrm{P}<0.05$ compared with the smoke-exposed (SM) group. C, sham smoke; SM, cigarette smoke; H, hemin + smoke; S, SnPP + smoke; HS, hemin + SnPP + smoke.

Hemin, as an inducer of HO-1, significantly upregulated HO-1 protein expression and attenuated pathological changes associated with emphysema in rats exposed to long-term cigarette smoke for 20 weeks. Previous studies have found that exposure to cigarette smoke extract (CSE) induces an increase in the expression of HO-1 in primary human lung fibroblasts, tracheal smooth muscle and alveolar epithelial cells (18-21). However, the increased expression levels of HO-1 did not provide sufficient protection against the inflammation and oxidative damage induced by CSE in order to inhibit the development of pulmonary emphysema. In addition, it has been demonstrated that a decreased HO-1 expression in the alveolar macrophages of patients with severe COPD may be related to a lower HO-1 inducibility by ROS, caused by HO-1 gene promoter polymorphisms (22-26).

As an HO-1 inhibitor, treatment with SnPP slightly decreased HO-1 expression compared to treatment with hemin plus SnPP; treatment with hemin + SnPP slightly increased HO-1 protein expression in the lungs, while HO-1 activity was inhibited, but it did not aggravate the adverse effects of exposure to smoke or increase the levels of inflammatory mediators induced by smoking. Although HO-1 activity is inhibited by
SnPP, the upregulation of HO-1 expression can have antiinflammatory and antioxidant effects (30).

Long-term exposure to smoke leads to inflammation and oxidative stress in the airways and lung tissue. In a previous study, exposure to smoke for 20 weeks significantly increased the levels of the pro-inflammatory cytokines, including TNF- $\alpha$, IL-1, IL-6, KC (CXCL1) and MCP-1 in the mouse lung tissue homogenate (30). Another study found that 3 days of mainstream smoke exposure induced an acute inflammatory response characterized by a neutrophilic influx, increased cytokine secretion (KC, TNF- $\alpha$, MIP-2, MIP-1 $\alpha$ and MCP-1) in a mouse model of COPD (31). The present study also found that smoking significantly increased the infiltration of inflammatory cells and the levels of cytokines, such as IL-17, TNF- $\alpha$, IL-8, MCP-1 and MIP- $2 \alpha$ and resulted in pathological changes associated with emphysema.

It has been found that the $\mathrm{CD} 4^{+} \mathrm{IL}-17^{+}$cell number positively correlates with airflow limitations and pathological changes and negatively correlates with FEV(1)\%p and FEV(1)/ FVC in patients with COPD. Increased $\mathrm{CD} 4^{+} \mathrm{IL}-17^{+}$cell numbers are closely related to chronic lung inflammation and may contribute to the pathogenesis of COPD (32). The admin- 
istration of IL-17 antibodies to mice exposed to cigarette smoke has been shown to reduce the number of neutrophils, the levels of IL-6 and IL-8, and neutrophil airway inflammation $(33,34)$. In a mouse model of elastase-induced pulmonary emphysema, the number of IL-17A ${ }^{+}$CD4 $\mathrm{T}$ cells and the levels of IL-17A, IL-1 $\beta, \mathrm{KC}$ and MIP-2 increased; however, the levels of these cytokines decreased in IL-17A-deficient mice following elastase treatment (35). IL-17 not only promotes the secretion of IL-8 (CXCL8) and TNF- $\alpha$, but also promotes the recruitment of neutrophils and monocytes, increases their functionality, extends their survival time in the lungs and thus causes lung inflammation $(5,32,34)$. Thus, IL-17A may be a more critical cytokine and may play an important role in the development of emphysema and pulmonary inflammation. However, a study of patients with COPD and smoke-induced COPD found elevated levels of IL-8 and decreased IL-17 levels in sputum and BALF. Time course experiments demonstrated that IL- 8 levels began to increase after 8 weeks of smoke exposure and IL-17 levels decreased after 10 weeks. Elevated IL-8 levels in patients with COPD appeared to be primarily derived from neutrophils and macrophages, and decreased IL-17 levels were mainly due to the decreased number of monocytes and damaged epithelial cells (36). These results were inconsistent as monocytes and cells other than Th17 cells can also secrete IL-17 and a decrease in the number of these cells may also affect the secretion of IL-17. Therefore, further studies evaluating the distribution of lymphocytes together with other cells are required.

Long-term treatment with hemin upregulated the protein expression of HO-1, reduced the infiltration of neutrophils and monocytes/macrophages, decreased the levels of proinflammatory cytokines, including IL-17, TNF- $\alpha$, IL-8, MCP-1 and MIP-2 $\alpha$, and inhibited pathological changes in the lungs, although the changes in the levels of these cytokines levels were not consistent. For example, the levels of IL-17 in the serum did not differ significantly among the experimental groups, possibly since IL-17 is mainly secreted by cells at sites of inflammation in lung tissue, and activated cells in the blood are recruited to the lungs. In a previous study, in a mouse model of non-eosinophilic asthma, the induction of HO-1 exerted anti-inflammatory effects by decreasing IL-17A levels via inhibition of Th17 cell differentiation. The HO-1 inhibitor, SnPP, reversed these effects and transfection with HO-1 siRNA abolished the effects of hemin on the induction of HO-1 in vivo (37). In a murine model of dextran sulfate sodium (DSS)-induced colitis, the upregulation of HO-1 by hemin attenuated IL-17 and Th17-related cytokine production and ameliorated experimental colitis (38). Thus, HO-1 may exert anti-inflammatory effects by inhibiting IL-17 and other cytokines, including TNF- $\alpha$, IL-8, MCP-1 and MIP- $2 \alpha$.

IL-10 is an important anti-inflammatory cytokine secreted by $\mathrm{B}$ cells, monocytes, regulatory $\mathrm{T}$ cells and others (39). Studies have found that the upregulation of HO-1 expression promotes the secretion of IL-10, thus promoting the antiinflammatory effects of IL-10 in several animal disease models, such as asthma and acute lung injury $(40,41)$. In addition, IL-10 induces the expression of HO-1, a downstream effector of IL-10, which plays an anti-inflammatory role in lipopolysaccharide-induced septic shock in mice $(42,43)$. These results suggest that HO-1 and IL-10 may form a positive loop to enhance the anti-inflammatory effect. The present study also found that the levels of IL-10 in BALF, serum and lung tissue homogenate were higher in the hemin group than those in the smoke-exposed group, indicating that hemin treatment exerts anti-inflammatory effects by promoting the secretion of IL-10.

Inflammation and oxidative stress, in combination, promote the development of emphysema. MDA is a major oxidative stress product produced by the attack of oxygen free radicals on polyunsaturated fatty acids and may be used as a marker to reflect the level of oxidative lipid damage in vivo. SOD is a indirect marker of the body's ability to eliminate oxygen free radicals. The concentrations of MDA and SOD often complement each other. In the present study, the MDA levels increased, whereas the SOD and GSH levels decreased in the smoke-exposed group, while hemin treatment decreased the MDA, and increased the SOD and GSH levels. These results indicated that hemin treatment improved the body's antioxidant capacity and reduced oxidative lipid damage.

The most important finding of the present study was the protective effects of HO-1 against the development of cigarette smoke-induced emphysema. HO-1 can alleviate lung pathological changes, possibly due to its capability to reduce the infiltration of inflammatory cells in lung tissue, the levels of inflammatory mediators and associated oxidative stress damage. However, HO-1 did not completely inhibit the inflammatory response and oxidative stress. A previous study found that long-term HO-1 upregulation induced by CoPP prevented smoke-induced $\mathrm{B}$ cell infiltrates and increased $\mathrm{CD} 4{ }^{+} \mathrm{CD} 25^{+}$Tregs, but had no effect on smoke-induced emphysema and the increase in inflammatory cells and cytokines in a mouse model (30). This result is not consistent with our study. This may be due to the differences in species selection in rodent models (rat vs. others) and treatment regimens (dose, treatment interval, or single or combinatorial pharmacological interventions). Thus, the exact role of HO-1 in the smokeinduced inflammatory response and oxidative stress remains to be elucidated.

In conclusion, our findings demonstrate that long-term HO-1 upregulation exerts a protective effect against the development of cigarette smoke-induced emphysema. A possible explanation for this effect of HO-1 on emphysema is its anti-inflammatory and antioxidant effects.

\section{Acknowledgements}

The authors would like to thank Dr Hongjuan Yang, Dr Fangrun Yuan, Dr Enguang Wang, Dr Junfang Wu and Mrs. Jiahui Zhao for their assistance in performing the smoke exposures and collecting experimental specimens. The present study was supported by the grants from the Health and Family Planning Commission of Shanxi Provice (2014039) and the Natural Science Foundation from the Science and Technology Department of Shanxi Province (project no. 2013011055-3).

\section{References}

1. Vestbo J,Hurd SS, Agustí AG,Jones PW,VogelmeierC,Anzueto A, Barnes PJ, Fabbri LM, Martinez FJ, Nishimura M, et al: Global strategy for the diagnosis, management, and prevention of chronic obstructive pulmonary disease: GOLD executive summary. Am J Respir Crit Care Med 187: 347-365, 2013. 
2. Barnes P.J: Chronic obstructive pulmonary disease. N Engl J Med 343: 269-280, 2000

3. Yoshida $T$ and Tuder RM: Pathobiology of cigarette smoke-induced chronic obstructive pulmonary disease. Physiol Rev 87: 1047-1082, 2007.

4. Tuder RM and Petrache I: Pathogenesis of chronic obstructive pulmonary disease. J Clin Invest 122: 2749-2755, 2012.

5. Zhang J, Chu S, Zhong X, Lao Q, He Z and Liang Y: Increased expression of $\mathrm{CD} 4^{+} \mathrm{IL}-17^{+}$cells in the lung tissue of patients with stable chronic obstructive pulmonary disease (COPD) and smokers. Int Immunopharmacol 15: 58-66, 2013.

6. Driscoll KE: Macrophage inflammatory proteins: biology and role in pulmonary inflammation. Exp Lung Res 20: 473-490, 1994.

7. Driscoll KE: TNFa and MIP-2: role in particle-induced inflammation and regulation by oxidative stress. Toxicol Lett 112-113: $177-183,2000$.

8. Traves SL, Culpitt SV, Russell RE, Barnes PJ and Donnelly LE: Increased levels of the chemokines GRO $\alpha$ and MCP-1 in sputum samples from patients with COPD. Thorax 57: 590-595, 2002.

9. de Boer WI, VK Alagappan and HS Sharma: Molecular mechanisms in chronic obstructive pulmonary disease: Potential targets for therapy. Cell Biochem Biophys 47: 131-148, 2007.

10. de Boer WI, Sont JK, van Schadewijk A, Stolk J, van Krieken JH and Hiemstra PS: Monocyte chemoattractant protein 1, interleukin 8, and chronic airways inflammation in COPD. J Pathol 190: 619-626, 2000.

11. Abraham NG: Therapeutic applications of human heme oxygenase gene transfer and gene therapy. Curr Pharm Des 9: 2513-2524, 2003.

12. Abraham NG and Kappas A: Pharmacological and clinical aspects of heme oxygenase. Pharmacol Rev 60: 79-127, 2008.

13. Chauveau C, Bouchet D, Roussel JC, Mathieu P, Braudeau C, Renaudin K, Tesson L, Soulillou JP, Iyer S and Buelow R: Gene transfer of heme oxygenase-1 and carbon monoxide delivery inhibit chronic rejection. Am J Transplant 2: 581-592, 2002.

14. Araujo JA, Meng L, Tward AD, Hancock WW, Zhai Y, Lee A, Ishikawa K, Iyer S, Buelow R, Busuttil RW, et al: Systemic rather than local heme oxygenase-1 overexpression improves cardiac allograft outcomes in a new transgenic mouse. J Immunol 171 1572-1580, 2003.

15. Ryter SW and Choi AM: Heme oxygenase-1: Redox regulation of a stress protein in lung and cell culture models. Antioxid Redox Signal 7: 80-91, 2005

16. Jia YX, Sekizawa K, Okinaga $S$, Lie R and Sasaki H: Role of heme oxygenase in pulmonary response to antigen challenge in sensitized rats in vivo. Int Arch Allergy Immunol 120: 141-145, 1999.

17. Wang XH, Wang K, Zhang F, Li XC, Li J, De W, Guo J, Qian XF and Fan Y: Heme oxygenase-1 alleviates ischemia/reperfusion injury in aged liver. World J Gastroenterol 11: 690-694, 2005.

18. Baglole CJ, Sime PJ and RP Phipps: Cigarette smoke-induced expression of heme oxygenase-1 in human lung fibroblasts is regulated by intracellular glutathione. Am J Physiol Lung Cell Mol Physiol 295: L624-L636, 2008.

19. Cheng SE, Lee IT, Lin CC, Kou YR and Yang CM: Cigarette smoke particle-phase extract induces HO-1 expression in human tracheal smooth muscle cells: role of the c-Src/NADPH oxidase/MAPK/Nrf2 signaling pathway. Free Radic Biol Med 48: 1410-1422, 2010

20. Fukano Y, Oishi M, Chibana F, Numazawa S and Yoshida T: Analysis of the expression of heme oxygenase-1 gene in human alveolar epithelial cells exposed to cigarette smoke condensate. J Toxicol Sci 31: 99-109, 2006

21. Maestrelli P, Messlemani E AH, De Fina O, Nowicki Y, Saetta M, Mapp C and Fabbri LM: Increased expression of heme oxygenase (HO)-1 in alveolar spaces and HO-2 in alveolar walls of smokers. Am J Respir Crit Care Med 164: 1508-1513, 2001.

22. Tsoumakidou M, Tzanakis N, Chrysofakis G and Siafakas NM: Nitrosative stress, heme oxygenase- 1 expression and airway inflammation during severe exacerbations of COPD. Chest 127 : 1911-1918, 2005

23. Maestrelli P, Páska C, Saetta M, Turato G, Nowicki Y, Monti S, Formichi B, Miniati M and Fabbri LM: Decreased haem oxygenase-1 and increased inducible nitric oxide synthase in the lung of severe COPD patients. Eur Respir J 21: 971-976, 2003.

24. Slebos DJ, Kerstjens HA, Rutgers SR, Kauffman HF, Choi AM and Postma DS: Haem oxygenase-1 expression is diminished in alveolar macrophages of patients with COPD. Eur Respir J 23 $652-653,2004$
25. Exner M, Minar E, Wagner O and Schillinger M: The role of heme oxygenase-1 promoter polymorphisms in human disease. Free Radic Biol Med 37: 1097-1104, 2004.

26. Lakhdar R, Denden S, Kassab A, Leban N, Knani J, Lefranc G, Miled A, Chibani JB and Khelil AH: Update in chronic obstructive pulmonary disease: Role of antioxidant and metabolizing gene polymorphisms. Exp Lung Res 37: 364-375, 2011.

27. Shinohara T, Kaneko T, Nagashima Y, Ueda A, Tagawa A and Ishigatsubo Y: Adenovirus-mediated transfer and overexpression of heme oxygenase $1 \mathrm{cDNA}$ in lungs attenuates elastase-induced pulmonary emphysema in mice. Hum Gene Ther 16: 318-327, 2005.

28. Li JQ, Wen Y, Zhao H, Liu ZL, Song MJ, Xu YJ and Zhang ZX: The effects of bilirubin concentration on laminin and epidermal growth factor expression in lung tissue and type II pneumocytes in smoking rats model. Zhonghua Nei Ke Za Zhi 44: 129-132, 2005 (In Chinese).

29. Thurlbeck WM: Measurement of pulmonary emphysema. Am Rev Respir Dis 95: 752-764, 1967.

30. Brandsma CA, Hylkema MN, van der Strate BW, Slebos DJ, Luinge MA, Geerlings M, Timens W, Postma DS and Kerstjens HA: Heme oxygenase-1 prevents smoke induced B-cell infiltrates: a role for regulatory T cells? Respir Res 9: 17, 2008.

31. John G, Kohse K, Orasche J, Reda A, Schnelle-Kreis J, Zimmermann R, Schmid O, Eickelberg O and Yildirim AÖ: The composition of cigarette smoke determines inflammatory cell recruitment to the lung in COPD mouse models. Clin Sci (Lond) 126: 207-221, 2014.

32. Vargas-Rojas MI, Ramírez-Venegas A, Limón-Camacho L, Ochoa L, Hernández-Zenteno R and Sansores RH: Increase of Th17 cells in peripheral blood of patients with chronic obstructive pulmonary disease. Respir Med 105: 1648-1654, 2011.

33. Shen N, Wang J, Zhao M,Pei F and He B: Anti-interleukin-17 antibodies attenuate airway inflammation in tobacco-smoke-exposed mice. Inhal Toxicol 23: 212-218, 2011.

34. Linden A and Adachi M: Neutrophilic airway inflammation and IL-17. Allergy 57: 769-775, 2002.

35. Kurimoto E, Miyahara N, Kanehiro A, Waseda K, Taniguchi A, Ikeda G, Koga H, Nishimori H, Tanimoto Y, Kataoka M, et al: IL-17A is essential to the development of elastase-induced pulmonary inflammation and emphysema in mice. Respir Res 14: 5, 2013.

36. Zhang X, Zheng H, Zhang H, Ma W, Wang F, Liu C and He S: Increased interleukin (IL)-8 and decreased IL-17 production in chronic obstructive pulmonary disease (COPD) provoked by cigarette smoke. Cytokine 56: 717-725, 2011.

37. Zhang Y, Zhang L, Wu J, Di C and Xia Z: Heme oxygenase-1 exerts a protective role in ovalbumin-induced neutrophilic airway inflammation by inhibiting Th17 cell-mediated immune response. J Biol Chem 288: 34612-34626, 2013.

38. Zhong W, Xia Z, Hinrichs D, Rosenbaum JT, Wegmann KW, Meyrowitz $\mathrm{J}$ and Zhang Z: Hemin exerts multiple protective mechanisms and attenuates dextran sulfate sodium-induced colitis. J Pediatr Gastroenterol Nutr 50: 132-139, 2010

39. Taylor A, Verhagen J, Blaser K, Akdis M and Akdis CA Mechanisms of immune suppression by interleukin-10 and transforming growth factor-beta: the role of T regulatory cells. Immunology 117: 433-442, 2006.

40. Inoue S, Suzuki M, Nagashima Y, Suzuki S, Hashiba T, Tsuburai T, Ikehara K, Matsuse T and Ishigatsubo Y: Transfer of heme oxygenase 1 cDNA by a replication-deficient adenovirus enhances interleukin 10 production from alveolar macrophages that attenuates lipopolysaccharide-induced acute lung injury in mice. Hum Gene Ther 12: 967-979, 2001.

41. Xia ZW, Xu LQ, Zhong WW, Wei JJ, Li NL, Shao J, Li YZ, Yu SC and Zhang ZL: Heme oxygenase-1 attenuates ovalbumin-induced airway inflammation by up-regulation of foxp3 T-regulatory cells, interleukin-10, and membrane-bound transforming growth factor-1. Am J Pathol 171: 1904-1914, 2007.

42. Lee TS and Chau LY: Heme oxygenase-1 mediates the anti-inflammatory effect of interleukin-10 in mice. Nat Med 8: 240-246, 2002

43. Drechsler Y, Dolganiuc A, Norkina O, Romics L, Li W, Kodys K, Bach FH, Mandrekar P and Szabo G: Heme oxygenase-1 mediates the anti-inflammatory effects of acute alcohol on IL-10 induction involving p38 MAPK activation in monocytes. J Immunol 177: 2592-2600, 2006. 